\title{
Exact Three-Dimensional Elasticity Solution for Buckling of Beam of a Circular Cross-Section*
}

\author{
Toshimi $\mathrm{TAKI}^{1 \dagger \dagger}$ and Kyohei KONDO${ }^{2)}$ \\ ${ }^{1)}$ Aerospace Company, Kawasaki Heavy Industries, Ltd., Kakamigahara, Gifu 504-8710, Japan \\ ${ }^{2)}$ Department of Aeronautics and Astronautics, The University of Tokyo, Tokyo 113-8656, Japan
}

\begin{abstract}
An exact three-dimensional elasticity solution for buckling of a circular cross-section beam with one end fixed and the other end free is obtained. A comparison is made using the approximate solution by Kardomateas and an exact two-dimensional solution by Chattopadhyay and Gu. The buckling loads of both exact solutions are in good agreement with each other, but the buckling load using the approximate solution is slightly higher than the exact solutions. The exact solutions support Engesser's formula rather than Haringx's formula for the buckling load of a beam with transverse shear effect.
\end{abstract}

Key Words: Buckling Analysis of Beam, Three-dimensional Elasticity, Transverse Shear Effect

\section{Nomenclature}

$$
\begin{aligned}
& a \text { : radius of circular cross-section beam } \\
& A \text { : cross sectional area of beam } \\
& C_{11}, C_{12}, \ldots \text { : components of stiffness matrix } \\
& C \text { : stiffness matrix } \\
& E, E_{L}, E_{T} \text { : Young's modulus } \\
& E_{r r}, E_{\theta \theta}, \Gamma_{r \theta}, \Gamma_{r z}, \ldots: \text { components of Green strain tensor } \\
& \boldsymbol{E} \text { : Green strain tensor } \\
& \boldsymbol{F} \text { : deformation gradient tensor } \\
& G, G_{L T}, G_{T T} \text { : shear modulus } \\
& \boldsymbol{G}_{r}, \boldsymbol{G}_{\theta}, \boldsymbol{G}_{z} \text { : base vectors after deformation in cylindrical } \\
& \text { coordinates } \\
& \boldsymbol{i}_{r}, \boldsymbol{i}_{\theta}, \boldsymbol{i}_{z} \text { : base vectors before deformation in cylindrical } \\
& \text { coordinates } \\
& \text { I: unit matrix } \\
& l, m, n \text { : components of normal vector of a surface } \\
& I_{n}(z) \text { : modified Bessel function of the first kind } \\
& k \text { : transverse shear factor } \\
& L \text { : length of beam } \\
& L^{\prime} \text { : effective column length }
\end{aligned}
$$

(C) 2017 The Japan Society for Aeronautical and Space Sciences *Presented at the 54th JSASS/JSME/JAXA Structures Conference, August 1-3, 2012, Kumamoto, Japan

Received 13 April 2016; final revision received 17 June 2016; accepted for publication 23 July 2016 .

†Corresponding author, taki_toshimi@khi.co.jp

\author{
cross-sectional area \\ $\boldsymbol{u}$ : displacement vector \\ $u, v, w$ : displacement components in cylindrical coor- \\ dinates \\ $v, v_{L T}, v_{T T}, \ldots:$ Poisson's ratio \\ $\rho$ : radius of gyration \\ $\sigma_{r r}, \sigma_{\theta \theta}, \tau_{r \theta}, \tau_{r z}, \ldots:$ components of Kirchhoff stress tensor \\ $\Sigma$ : Kirchhoff stress tensor in cylindrical coordi- \\ nates \\ Subscripts
}

0 : before buckling

1: after buckling

E: Euler

En: Engesser

$H$ : Haringx

$K$ : Kardomateas

TK: Taki and Kondo

\section{Introduction}

The study of elastic beam buckling has a long history and many studies are still being conducted in this field. The effect of transverse shear stiffness on the buckling load of a beam is an important topic. As referred to in Timoshenko and Gere, ${ }^{1)}$ buckling load formulas by Engesser and Haringx are proposed for a beam with transverse shear deformation. The Engesser formula was proposed for beams, and the Haringx formula was first proposed for buckling loads of coil springs and rubber rods. There has been a controversy about which formula is suitable for beams with low transverse shear stiffness, such as lattice beams and sandwich beams.

The Engesser and Heringx formulas were derived using one-dimensional theories, and they are based on different assumptions in the definition of axial and shear forces acting on the beam cross-section. Two-dimensional analysis or threedimensional analysis of the beam is necessary to judge which is correct. Chattopadhyay and $\mathrm{Gu}^{2)}$ derived an exact twodimensional solution for the elastic buckling of a rectangular orthotropic plate. They did not mention the Engesser and 
Haringx formulas in their paper. Kardomateas ${ }^{3)}$ conducted three-dimensional elasticity analysis for buckling of a beam with a circular cross-section. He calculated the buckling load of a beam made of orthotropic material and compared the result with buckling loads using the Engesser and Haringx formulas. He concluded that his elasticity solution is closer to the Haringx formula than the Engesser formula. Kardomateas' paper encourages proponents of the Haringx formula, and this is a reason why the Haringx formula was recognized as the correct formula.

Bažant ${ }^{4)}$ explained that the difference between the Engesser and Haringx formulas is attributed to different finite strain measures, and the Haringx formula is more convenient than the Engesser formula. Bažant changed his position to support the Engesser formula in the papers on buckling of a sandwich beam. ${ }^{5,6)} \mathrm{He}$ concluded that analysis and buckling test data of the sandwich beam support that the Engesser formula is correct.

There still exist some proponents of the Haringx formula, ${ }^{3,7)}$ but it may be concluded that the controversy has ended. ${ }^{8,9)}$ The conclusion is that the Engesser formula is applicable to beams with weak transverse shear stiffness including lattice beams and sandwich beams, and the Haringx formula is applicable only to coil springs and rubber bearings.

This paper presents an exact solution of three-dimensional elasticity for buckling of a circular cross-section beam. The exact three-dimensional solution is compared to an exact two-dimensional solution proposed by Chattopadhyay and $\mathrm{Gu}$. Then, the buckling loads of beams made of orthotropic material are compared to the buckling loads derived using the Engesser and Haringx formulas. It is shown in this paper that the solution by Kardomateas is an approximate solution, and the exact solution supports applicability of the Engesser formula to the buckling load of beams with a transverse shear effect.

\section{Formulation}

Compression buckling of a circular cross-section beam with one end fixed and the other end free (see Fig. 1) is analyzed using a three-dimensional elasticity theory.

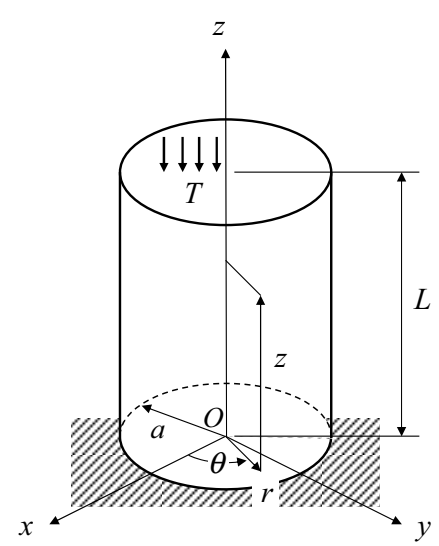

Fig. 1. Circular cross-section beam with one end fixed and the other end free.

\subsection{Governing equations}

Point A defined by position vector $\boldsymbol{r}$ in the cylindrical coordinates moves to a new position $\mathrm{A}^{\prime}$, which has a position vector $\boldsymbol{R}$ as shown in Fig. 2. Position vector $\boldsymbol{R}$ is expressed using the base vector before deformation and displacement components.

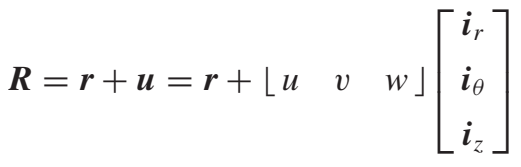

Base vectors after deformation are,

$$
\left[\begin{array}{c}
\boldsymbol{G}_{r} \\
\boldsymbol{G}_{\theta} \\
\boldsymbol{G}_{z}
\end{array}\right]=\left[\begin{array}{c}
\frac{\partial}{\partial r} \\
\frac{\partial}{r \partial \theta} \\
\frac{\partial}{\partial z}
\end{array}\right] \boldsymbol{R}=\boldsymbol{F}^{\mathrm{T}}\left[\begin{array}{c}
\boldsymbol{i}_{r} \\
\boldsymbol{i}_{\theta} \\
\boldsymbol{i}_{z}
\end{array}\right]
$$

where $\boldsymbol{F}$ is the deformation gradient tensor.

$$
\boldsymbol{F}^{\mathrm{T}}=\left[\begin{array}{ccc}
1+\frac{\partial u}{\partial r} & \frac{\partial v}{\partial r} & \frac{\partial w}{\partial r} \\
\frac{\partial u}{r \partial \theta}-\frac{v}{r} & 1+\frac{\partial v}{r \partial \theta}+\frac{u}{r} & \frac{\partial w}{r \partial \theta} \\
\frac{\partial u}{\partial z} & \frac{\partial v}{\partial z} & 1+\frac{\partial w}{\partial z}
\end{array}\right]
$$

Stress vectors $\boldsymbol{P}_{r}, \boldsymbol{P}_{\theta}, \boldsymbol{P}_{z}$ are expressed by the Kirchhoff stress tensor $\Sigma$ as follows.

$$
\left[\begin{array}{c}
\boldsymbol{P}_{r} \\
\boldsymbol{P}_{\theta} \\
\boldsymbol{P}_{z}
\end{array}\right]=\left[\begin{array}{ccc}
\sigma_{r r} & \tau_{r \theta} & \tau_{r z} \\
& \sigma_{\theta \theta} & \tau_{\theta z} \\
\text { Sym. } & & \sigma_{z z}
\end{array}\right]\left[\begin{array}{c}
\boldsymbol{G}_{r} \\
\boldsymbol{G}_{\theta} \\
\boldsymbol{G}_{z}
\end{array}\right]=\Sigma \boldsymbol{F}^{\mathrm{T}}\left[\begin{array}{c}
\boldsymbol{i}_{r} \\
\boldsymbol{i}_{\theta} \\
\boldsymbol{i}_{z}
\end{array}\right]
$$

The equilibrium equation is,

$$
\begin{aligned}
& \left\lfloor\begin{array}{lll}
\frac{\partial}{\partial r} & \frac{\partial}{r \partial \theta} & \frac{\partial}{\partial z}
\end{array}\right\rfloor\left[\Sigma \boldsymbol{F}^{\mathrm{T}}\right] \\
& +\frac{1}{r}\left\lfloor\sigma_{r r} \quad \sigma_{\theta \theta} \quad \tau_{r z} \quad \tau_{\theta z} \quad \tau_{r \theta}\right\rfloor \boldsymbol{H}=0
\end{aligned}
$$

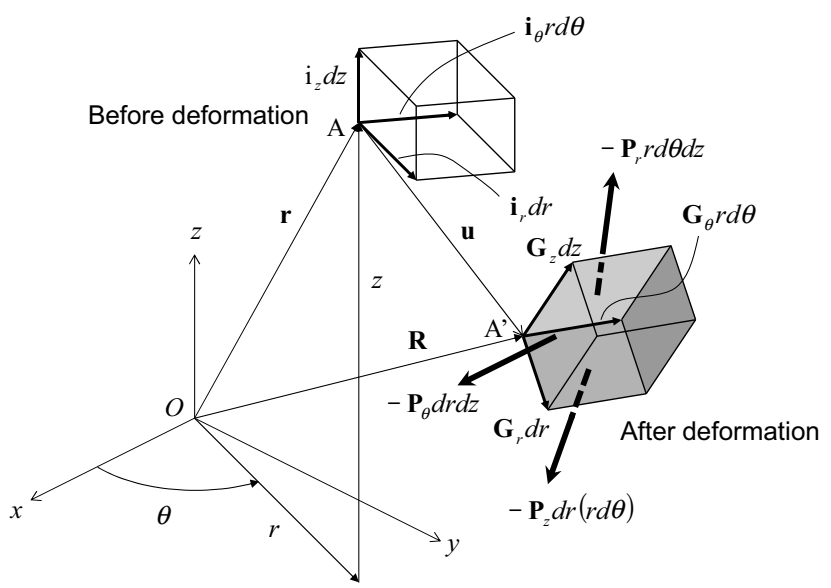

Fig. 2. Infinitesimal parallelepiped and stress vectors in cylindrical coordinates. 
where

$$
\boldsymbol{H}=\left[\begin{array}{ccc}
1+\frac{\partial u}{\partial r} & \frac{\partial v}{\partial r} & \frac{\partial w}{\partial r} \\
-\left(1+\frac{\partial v}{r \partial \theta}+\frac{u}{r}\right) & \frac{\partial u}{r \partial \theta}-\frac{v}{r} & 0 \\
\frac{\partial u}{\partial z} & \frac{\partial v}{\partial z} & 1+\frac{\partial w}{\partial z} \\
-\frac{\partial v}{\partial z} & \frac{\partial u}{\partial z} & 0 \\
-\frac{\partial v}{\partial r}+\frac{\partial u}{r \partial \theta}-\frac{v}{r} & 2+\frac{\partial u}{\partial r}+\frac{\partial v}{r \partial \theta}+\frac{u}{r} & \frac{\partial w}{r \partial \theta}
\end{array}\right]
$$

The Green strain tensor is expressed as

$$
\boldsymbol{E}=\frac{1}{2}\left(\boldsymbol{F}^{\mathrm{T}} \boldsymbol{F}-\boldsymbol{I}\right)=\left[\begin{array}{ccc}
E_{r r} & \Gamma_{r \theta} / 2 & \Gamma_{r z} / 2 \\
& E_{\theta \theta} & \Gamma_{\theta z} / 2 \\
\text { Sym. } & & E_{z z}
\end{array}\right]
$$

where

$$
\begin{aligned}
& E_{r r}=\frac{\partial u}{\partial r}+\frac{1}{2}\left\{\left(\frac{\partial u}{\partial r}\right)^{2}+\left(\frac{\partial v}{\partial r}\right)^{2}+\left(\frac{\partial w}{\partial r}\right)^{2}\right\} \\
& \Gamma_{r \theta}=\frac{\partial u}{r \partial \theta}-\frac{v}{r}+\frac{\partial v}{\partial r}+\left(\frac{\partial u}{\partial r}\right)\left(\frac{\partial u}{r \partial \theta}-\frac{v}{r}\right) \\
& +\left(\frac{\partial v}{\partial r}\right)\left(\frac{\partial v}{r \partial \theta}+\frac{u}{r}\right)+\left(\frac{\partial w}{\partial r}\right)\left(\frac{\partial w}{r \partial \theta}\right) \\
& \Gamma_{r z}=\frac{\partial u}{\partial z}+\frac{\partial w}{\partial r}+\left(\frac{\partial u}{\partial r}\right)\left(\frac{\partial u}{\partial z}\right)+\left(\frac{\partial v}{\partial r}\right)\left(\frac{\partial v}{\partial z}\right) \\
& +\left(\frac{\partial w}{\partial r}\right)\left(\frac{\partial w}{\partial z}\right) \\
& E_{\theta \theta}=\frac{\partial v}{r \partial \theta}+\frac{u}{r} \\
& +\frac{1}{2}\left\{\left(\frac{\partial u}{r \partial \theta}-\frac{v}{r}\right)^{2}+\left(\frac{\partial v}{r \partial \theta}+\frac{u}{r}\right)^{2}+\left(\frac{\partial w}{r \partial \theta}\right)^{2}\right\} \\
& \Gamma_{\theta z}=\frac{\partial v}{\partial z}+\frac{\partial w}{r \partial \theta}+\left(\frac{\partial u}{r \partial \theta}-\frac{v}{r}\right)\left(\frac{\partial u}{\partial z}\right)+\left(\frac{\partial v}{r \partial \theta}+\frac{u}{r}\right)\left(\frac{\partial v}{\partial z}\right) \\
& +\left(\frac{\partial w}{r \partial \theta}\right)\left(\frac{\partial w}{\partial z}\right) \\
& E_{z z}=\frac{\partial w}{\partial z}+\frac{1}{2}\left\{\left(\frac{\partial u}{\partial z}\right)^{2}+\left(\frac{\partial v}{\partial z}\right)^{2}+\left(\frac{\partial w}{\partial z}\right)^{2}\right\}
\end{aligned}
$$

The stress-strain relationship for transversely isotropic material is written as,

$$
\begin{aligned}
& \Sigma_{(i)}=\left[\begin{array}{c}
\sigma_{r r} \\
\sigma_{\theta \theta} \\
\sigma_{z z} \\
\tau_{\theta z} \\
\tau_{z r} \\
\tau_{r \theta}
\end{array}\right] \\
& =\left[\begin{array}{cccccc}
C_{11} & C_{12} & C_{13} & 0 & 0 & 0 \\
& C_{11} & C_{13} & 0 & 0 & 0 \\
& & C_{33} & 0 & 0 & 0 \\
& & & C_{55} & 0 & 0 \\
& & & & C_{55} & 0 \\
\text { Sym. } & & & & & \frac{C_{11}-C_{12}}{2}
\end{array}\right]\left[\begin{array}{c}
E_{r r} \\
E_{\theta \theta} \\
E_{z z} \\
\Gamma_{\theta z} \\
\Gamma_{z r} \\
\Gamma_{r \theta}
\end{array}\right]
\end{aligned}
$$

$$
=\boldsymbol{C E}_{(i)}
$$

where $\Sigma_{(i)}$ and $\boldsymbol{E}_{(i)}$ are Kirchhoff stress and Green strain expressed in vector form, respectively.

The stiffness matrix $\boldsymbol{C}$ is expressed using engineering elastic properties as follows.

$\boldsymbol{E}_{(i)}=\boldsymbol{C}^{-1} \Sigma_{(i)}$

$$
=\left[\begin{array}{cccccc}
\frac{1}{E_{T}} & -\frac{v_{T T}}{E_{T}} & -\frac{v_{L T}}{E_{L}} & 0 & 0 & 0 \\
-\frac{v_{T T}}{E_{T}} & \frac{1}{E_{T}} & -\frac{v_{L T}}{E_{L}} & 0 & 0 & 0 \\
-\frac{v_{T L}}{E_{T}} & -\frac{v_{T L}}{E_{T}} & \frac{1}{E_{L}} & 0 & 0 & 0 \\
& & \frac{1}{G_{L T}} & 0 & 0 \\
\text { Sym. } & & & \frac{1}{G_{L T}} & 0 \\
& & & & \frac{1}{G_{T T}}
\end{array}\right] \Sigma_{(i)}
$$

The definition of the engineering elastic properties of the transversely isotropic material is shown in Fig. 3. The following relationship exists between the properties.

$$
\frac{v_{L T}}{E_{L}}=\frac{v_{T L}}{E_{T}}, \quad G_{T T}=\frac{E_{T}}{2\left(1+v_{T T}\right)}
$$

The geometrical boundary condition is,

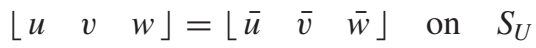

where $S_{U}$ is the surface on which displacements are specified.

The mechanical boundary condition is,

$\lfloor l l m b n\rfloor \Sigma \boldsymbol{F}^{\mathrm{T}}\left[\begin{array}{c}\boldsymbol{i}_{r} \\ \boldsymbol{i}_{\theta} \\ \boldsymbol{i}_{z}\end{array}\right]=\left\lfloor\begin{array}{lll}\bar{t}_{r} & \bar{t}_{\theta} & \bar{t}_{z}\end{array}\right\rfloor\left[\begin{array}{c}\boldsymbol{i}_{r} \\ \boldsymbol{i}_{\theta} \\ \boldsymbol{i}_{z}\end{array}\right] \quad$ on $\quad S_{F}$ 


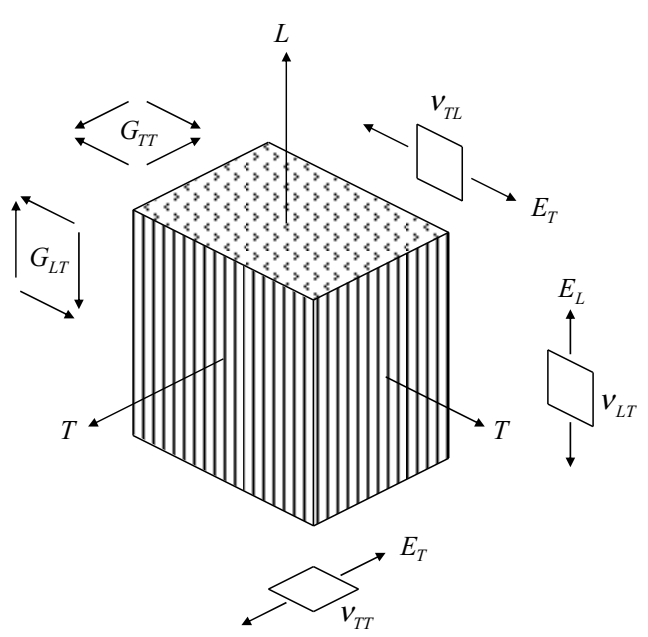

Fig. 3. Definition of elastic properties of the transversely isotropic material.

where $S_{F}$ is the surface on which the surface forces are specified.

\subsection{Pre-buckling equations and buckling equations}

Deformation is expressed by the sum of the pre-buckling

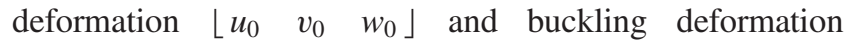
$\alpha\left\lfloor u_{1} \quad v_{1} \quad w_{1}\right\rfloor$.

$\left\lfloor\begin{array}{lll}u & v & w\end{array}=\left\lfloor\begin{array}{lll}u_{0} & v_{0} & w_{0}\end{array}\right\rfloor+\alpha\left\lfloor u_{1} \quad v_{1} \quad w_{1}\right\rfloor\right.$

From Eqs. (7) and (9), we obtain,

$$
\begin{gathered}
\boldsymbol{E}=\boldsymbol{E}_{0}+\alpha \boldsymbol{E}_{1} \\
\Sigma_{(i)}=\boldsymbol{C}\left(\boldsymbol{E}_{(i) 0}+\alpha \boldsymbol{E}_{(i) 1}\right)=\Sigma_{(i) 0}+\alpha \Sigma_{(i) 1}
\end{gathered}
$$

From Eqs. (3) and (6),

$$
\boldsymbol{F}^{\mathrm{T}}=\boldsymbol{F}_{0}^{\mathrm{T}}+\alpha \boldsymbol{F}_{1}^{\mathrm{T}}, \quad \boldsymbol{H}=\boldsymbol{H}_{0}+\alpha \boldsymbol{H}_{1}
$$

Then, the equilibrium equation is expressed as follows.

$$
\begin{aligned}
& \left\lfloor\frac{\partial}{\partial r} \frac{\partial}{r \partial \theta} \frac{\partial}{\partial z}\right\rfloor\left(\Sigma_{0} \boldsymbol{F}_{0}^{\mathrm{T}}\right)+\frac{1}{r}\left\lfloor\begin{array}{lllll}
\sigma_{r r 0} & \sigma_{\theta \theta 0} & \tau_{r z 0} & \tau_{\theta z 0} & \tau_{r \theta 0}
\end{array}\right\rfloor \boldsymbol{H}_{0}
\end{aligned}
$$

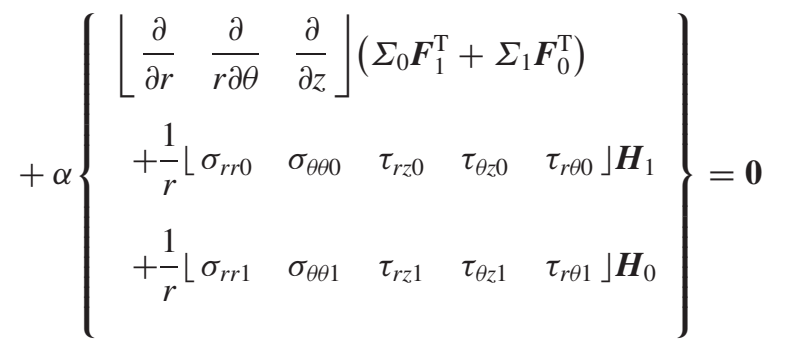

The boundary conditions are expressed as follows.

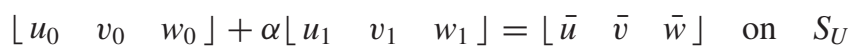

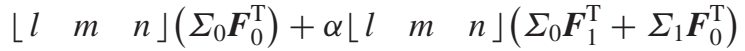

$$
\begin{aligned}
& =\left\lfloor\begin{array}{lll}
\bar{t}_{r} & \bar{t}_{\theta} & \bar{t}_{z}
\end{array} \text { on } \quad S_{F}\right.
\end{aligned}
$$

\section{Buckling Analysis}

A buckling analysis is conducted according to the procedure given in Kardomateas. ${ }^{3)}$ The difference is that we consider pre-buckling deformation while Kardomateas did not.

\subsection{Pre-buckling state}

The stress condition under uniform compression as shown in Fig. 1, is expressed as follows.

$$
\Sigma_{0}=\left[\begin{array}{ccc}
0 & 0 & 0 \\
0 & 0 & 0 \\
0 & 0 & -p
\end{array}\right]
$$

From the mechanical boundary condition at $z=L$, we obtain,

$$
p\left(1+\frac{\partial w_{0}}{\partial z}\right)=T
$$

where $T$ is the compressive stress defined as total force on the upper end divided by the initial (undeformed) cross-sectional area.

Deformation before buckling is expressed as follows.

$$
\begin{aligned}
& \frac{\partial u_{0}}{\partial r}=\frac{u_{0}}{r}=-1+\sqrt{1-2 c_{1} p} \\
& \frac{\partial w_{0}}{\partial z}=-1+\sqrt{1-2 c_{2} p}
\end{aligned}
$$

where

$$
\left[\begin{array}{l}
c_{1} \\
c_{2}
\end{array}\right]=\frac{1}{\left|\begin{array}{lll}
C_{11} & C_{12} & C_{13} \\
C_{12} & C_{11} & C_{13} \\
C_{13} & C_{13} & C_{33}
\end{array}\right|}\left[\begin{array}{c}
\left|\begin{array}{ll}
C_{12} & C_{13} \\
C_{11} & C_{13}
\end{array}\right| \\
\ldots \ldots \ldots \ldots \ldots \\
\left|\begin{array}{ll}
C_{11} & C_{12} \\
C_{12} & C_{11}
\end{array}\right|
\end{array}\right]
$$

Kardomateas did not consider these equations.

\subsection{Buckling state}

Substituting Eq. (21) for the equilibrium equation (Eq. (18)), we obtain the following equation.

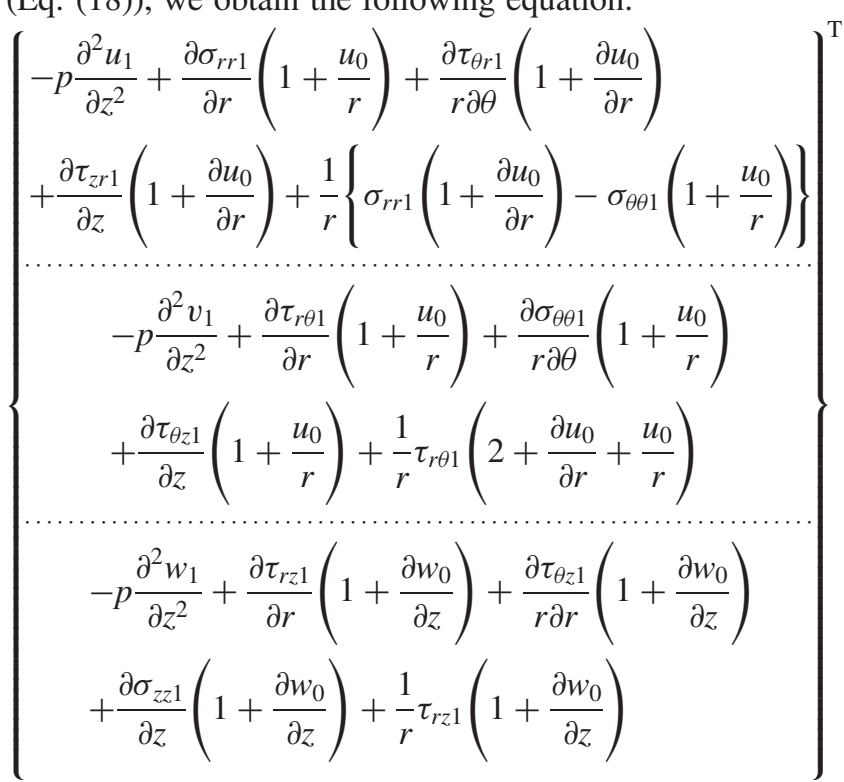




$$
=\left\{\begin{array}{l}
0 \\
0 \\
0
\end{array}\right\}^{\mathrm{T}}
$$

From the geometrical boundary condition (Eq. (19)),

$$
\left\lfloor\begin{array}{lll}
u_{1} & v_{1} & w_{1}
\end{array}\right\rfloor=\left\lfloor\begin{array}{lll}
0 & 0 & 0
\end{array}\right\rfloor \quad \text { on } \quad S_{U}
$$

From the mechanical boundary condition (Eq. (20)),

$$
\begin{aligned}
& \left\lfloor\begin{array}{lll}
l & m & n
\end{array}\right\rfloor \\
& \times\left[\begin{array}{c:c:c}
\sigma_{r r 1}\left(1+\frac{\partial u_{0}}{\partial r}\right) & \tau_{r \theta 1}\left(1+\frac{u_{0}}{r}\right) & \tau_{r z 1}\left(1+\frac{\partial w_{0}}{\partial z}\right) \\
\hdashline \tau_{\theta r 1}\left(1+\frac{\partial u_{0}}{\partial r}\right) & \sigma_{\theta \theta 1}\left(1+\frac{u_{0}}{r}\right) & \tau_{\theta z 1}\left(1+\frac{\partial w_{0}}{\partial z}\right) \\
\hdashline-p \frac{\partial u_{1}}{\partial z} & -p \frac{\partial v_{1}}{\partial z} & -p \frac{\partial w_{1}}{\partial z} \\
\hdashline \tau_{z r 1}\left(1+\frac{\partial u_{0}}{\partial r}\right) & +\tau_{z \theta 1}\left(1+\frac{u_{0}}{r}\right) & +\sigma_{z z 1}\left(1+\frac{\partial w_{0}}{\partial z}\right)
\end{array}\right] \\
& =\left\lfloor\begin{array}{lll}
0 & 0 & 0
\end{array}\right\rfloor \quad \text { on } \quad S_{F}
\end{aligned}
$$

From Eq. (27), the condition for the external force free on the upper end is expressed as follows.

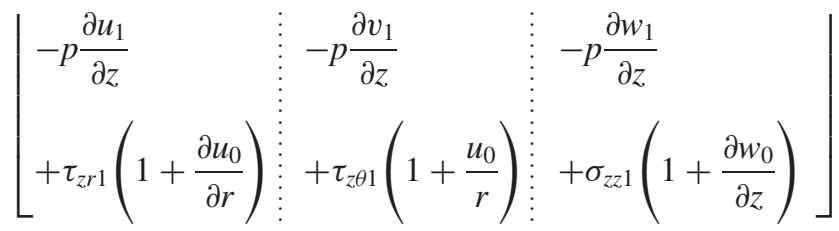

$$
\begin{aligned}
& =\left\lfloor\begin{array}{lll}
0 & 0 & 0
\end{array}\right\rfloor \quad \text { at } \quad z=L
\end{aligned}
$$

From the condition for the external force free on the surface of the beam, the following stresses are zero.

$$
\sigma_{r r 1}=0, \quad \tau_{r \theta 1}=0, \quad \tau_{r z 1}=0 \quad \text { on } \quad r=a
$$

From Eq. (16),

$$
\Sigma_{(i) 1}=\boldsymbol{C} \boldsymbol{E}_{(i) 1}
$$

Substituting Eq. (30) for Eq. (25), the following equations are obtained. These equations (Eq. (31)) correspond to Eq. (11) in Kardomateas. ${ }^{3)}$

$$
\begin{gathered}
C_{11}\left(\frac{\partial^{2} u_{1}}{\partial r^{2}}+\frac{\partial u_{1}}{r \partial r}-\frac{u_{1}}{r^{2}}\right)+\frac{C_{11}-C_{12}}{2}\left(\frac{\partial^{2} u_{1}}{r^{2} \partial \theta^{2}}\right) \\
+\left\{C_{55}-p /\left(1+\frac{u_{0}}{r}\right)^{2}\right\}\left(\frac{\partial^{2} u_{1}}{\partial z^{2}}\right) \\
+\frac{C_{11}+C_{12}}{2} \frac{\partial}{\partial r}\left(\frac{1}{r} \frac{\partial v_{1}}{\partial \theta}\right)-\left(C_{11}-C_{12}\right)\left(\frac{\partial v_{1}}{r^{2} \partial \theta}\right)
\end{gathered}
$$

$$
+\left(\frac{1+\frac{\partial w_{0}}{\partial z}}{1+\frac{u_{0}}{r}}\right)\left(C_{13}+C_{55}\right)\left(\frac{\partial^{2} w_{1}}{\partial r \partial z}\right)=0
$$$$
\frac{C_{11}-C_{12}}{2}\left(\frac{\partial^{2} v_{1}}{\partial r^{2}}+\frac{\partial v_{1}}{r \partial r}-\frac{v_{1}}{r^{2}}\right)+C_{11}\left(\frac{\partial^{2} v_{1}}{r^{2} \partial \theta^{2}}\right)
$$$$
+\left\{C_{55}-p /\left(1+\frac{u_{0}}{r}\right)^{2}\right\}\left(\frac{\partial^{2} v_{1}}{\partial z^{2}}\right)
$$$$
+\frac{\partial}{r \partial \theta}\left[\begin{array}{c}
\frac{C_{11}+C_{12}}{2}\left(\frac{\partial u_{1}}{\partial r}+\frac{u_{1}}{r}\right)+\left(C_{11}-C_{12}\right) \frac{u_{1}}{r} \\
+\left(\frac{1+\frac{\partial w_{0}}{\partial z}}{1+\frac{u_{0}}{r}}\right)\left(C_{13}+C_{55}\right)\left(\frac{\partial w_{1}}{\partial z}\right)
\end{array}\right]=0
$$$$
C_{55}\left(\frac{\partial^{2} w_{1}}{\partial r^{2}}+\frac{\partial w_{1}}{r \partial r}+\frac{\partial^{2} w_{1}}{r^{2} \partial \theta^{2}}\right)
$$$$
+\left\{C_{33}-p /\left(1+\frac{\partial w_{0}}{\partial z}\right)^{2}\right\}\left(\frac{\partial^{2} w_{1}}{\partial z^{2}}\right)
$$$$
+\left(\frac{1+\frac{u_{0}}{r}}{1+\frac{\partial w_{0}}{\partial z}}\right)\left(C_{13}+C_{55}\right) \frac{\partial}{\partial z}\left(\frac{\partial u_{1}}{\partial r}+\frac{u_{1}}{r}+\frac{\partial v_{1}}{r \partial \theta}\right)=0
$$

Following the procedure of Kardomateas, ${ }^{3)}$ we consider two groups of solutions for the equilibrium equation (31). Rigid-body displacement is not considered here because rigid-body displacement does not affect the buckling load. The final buckling solution is the superposition of the two groups of the solutions.

\subsubsection{First group solution}

The following functions satisfy the equilibrium equation, Eq. (31).

$$
u_{1}=\frac{\partial \phi}{\partial r}, \quad v_{1}=\frac{\partial \phi}{r \partial \theta}, \quad w_{1}=k \frac{\partial \phi}{\partial z}
$$

where $k$ is a constant. Substituting Eq. (32) for Eq. (31), we obtain,

$$
\begin{aligned}
& C_{11}\left(\frac{\partial^{2} \phi}{\partial r^{2}}+\frac{1}{r} \frac{\partial \phi}{\partial r}+\frac{1}{r^{2}} \frac{\partial^{2} \phi}{\partial \theta^{2}}\right) \\
& +\left\{C_{55}+k\left(\frac{1+\frac{\partial w_{0}}{\partial z}}{1+\frac{u_{0}}{r}}\right)\left(C_{13}+C_{55}\right)-p /\left(1+\frac{u_{0}}{r}\right)^{2}\right\} \\
& \quad \times\left(\frac{\partial^{2} \phi}{\partial z^{2}}\right)=0
\end{aligned}
$$




$$
\begin{gathered}
\left\{k C_{55}+\left(\frac{1+\frac{u_{0}}{r}}{1+\frac{\partial w_{0}}{\partial z}}\right)\left(C_{13}+C_{55}\right)\right\}\left(\frac{\partial^{2} \phi}{\partial r^{2}}+\frac{1}{r} \frac{\partial \phi}{\partial r}+\frac{1}{r^{2}} \frac{\partial^{2} \phi}{\partial \theta^{2}}\right) \\
+k\left\{C_{33}-p /\left(1+\frac{\partial w_{0}}{\partial z}\right)^{2}\right\}\left(\frac{\partial^{2} \phi}{\partial z^{2}}\right)=0
\end{gathered}
$$

From Eqs. (33) and (34),

$$
\frac{\partial^{2} \phi_{i}}{\partial r^{2}}+\frac{1}{r} \frac{\partial \phi_{i}}{\partial r}+\frac{1}{r^{2}} \frac{\partial^{2} \phi_{i}}{\partial \theta^{2}}+s_{i}^{2} \frac{\partial^{2} \phi_{i}}{\partial z^{2}}=0
$$

where $s_{i}$ is the solution of the following equation. This equation corresponds to Eq. (14b) in Kardomateas. ${ }^{3)}$

$$
\begin{aligned}
& C_{11} C_{55} s^{4} \\
& +\left[\begin{array}{l}
\left(C_{13}+C_{55}\right)^{2}-C_{11}\left\{C_{33}-p /\left(1+\frac{\partial w_{0}}{\partial z}\right)^{2}\right\} \\
-C_{55}\left\{C_{55}-p /\left(1+\frac{u_{0}}{r}\right)^{2}\right\}
\end{array} s^{2}\right. \\
& +\left\{C_{55}-p /\left(1+\frac{u_{0}}{r}\right)^{2}\right\}\left\{C_{33}-p /\left(1+\frac{\partial w_{0}}{\partial z}\right)^{2}\right\}
\end{aligned}
$$

Then, $k_{i}$ are expressed with $s_{i}$ as follows. This equation corresponds to Eq. (14c) in Kardomateas. ${ }^{3)}$

$$
k_{i}=\frac{C_{11} s_{i}^{2}-C_{55}+\frac{p}{\left(1+\frac{u_{0}}{r}\right)^{2}}}{\frac{1+\frac{\partial w_{0}}{\partial z}}{1+\frac{u_{0}}{r}}\left(C_{13}+C_{55}\right)}(i=1,2)
$$

The following function is considered as the solution of Eq. (35).

$$
\phi_{i}(r, \theta, z)=Z(z) A_{i}(\lambda r) \cos \theta \quad(i=1,2)
$$

Assuming Eqs. (39) and (40), we obtain Eq. (41) from Eq. (35).

$$
\begin{gathered}
Z(z)=\cos \lambda z \\
\lambda r=\rho \\
\frac{\mathrm{d}^{2} A_{i}(\rho)}{\mathrm{d}\left(s_{i} \rho\right)^{2}}+\frac{1}{\left(s_{i} \rho\right)} \frac{\mathrm{d} A_{i}(\rho)}{\mathrm{d}\left(s_{i} \rho\right)}-\left(1+\frac{1}{\left(s_{i} \rho\right)^{2}}\right) A_{i}(\rho)=0
\end{gathered}
$$

The solution of Eq. (41) is as follows.

$$
A_{i}(\rho)=C_{i} I_{1}\left(s_{i} \rho\right)
$$

where $I_{1}\left(s_{i} \rho\right)$ is the modified Bessel function of the first order of the first kind. Finally we have the following expression for $\phi_{i}$.

$$
\phi_{i}(r, \theta, z)=C_{i}(\cos \lambda z) I_{1}\left(s_{i} \rho\right) \cos \theta \quad(i=1,2)
$$

\subsubsection{Second group solution}

The following functions satisfy the equilibrium equation, Eq. (31).

$$
u_{1}=\frac{\partial \psi}{r \partial \theta}, \quad v_{1}=-\frac{\partial \psi}{\partial r}, \quad w_{1}=0
$$

Substituting Eq. (44) for Eq. (31),

$$
\frac{\partial^{2} \psi}{\partial r^{2}}+\frac{\partial \psi}{r \partial \theta}+\frac{1}{r^{2}} \frac{\partial^{2} \psi}{\partial \theta^{2}}+q^{2} \frac{\partial^{2} \psi}{\partial z^{2}}=0
$$

where $q$ is expressed as follows.

$$
q^{2}=\frac{C_{55}-\frac{p}{\left(1+\frac{u_{0}}{r}\right)^{2}}}{\frac{C_{11}-C_{12}}{2}}
$$

The following function is considered as the solution of Eq. (45).

$$
\psi(r, \theta)=Z(z) B(\lambda r) \sin \theta
$$

From Eqs. (45) and (47),

$$
\frac{\mathrm{d}^{2} B(\rho)}{\mathrm{d}(q \rho)^{2}}+\frac{1}{q \rho} \frac{\mathrm{d} B(\rho)}{\mathrm{d}(q \rho)}-\left(1+\frac{1}{(q \rho)^{2}}\right) B(\rho)=0
$$

The solution of Eq. (48) is as follows.

$$
B(\rho)=C_{0} I_{1}(q \rho)=0
$$

Substituting Eqs. (39) and (49) for Eq. (47), we obtain $\psi$.

$$
\psi(r, \theta, z)=C_{0} \cos \lambda z I_{1}(q \rho) \sin \theta
$$

Deflections are calculated from Eqs. (32) and (44),

$$
\begin{aligned}
& u_{1}=\frac{\partial \phi_{i}}{\partial r}+\frac{\partial \psi}{r \partial \theta} \\
& v_{1}=\frac{\partial \phi_{i}}{r \partial \theta}-\frac{\partial \psi}{\partial r} \\
& w_{1}=k \frac{\partial \phi_{i}}{\partial z}
\end{aligned}
$$

From the boundary condition at the upper end (Eq. (28)),

$$
-p \frac{\partial w_{1}}{\partial z}+\sigma_{z z 1}\left(1+\frac{\partial w_{0}}{\partial z}\right)=0 \quad \text { at } \quad z=L
$$

It is shown that $\partial w_{1} / \partial z$ and $\sigma_{z z 1}$ in Eq. (52) are proportional to $\cos \lambda z$, and Eq. (52) is satisfied with the following condition.

$$
\lambda=\frac{\pi}{2 L}
$$

Using the above equations, the stresses are expressed as follows. 


$$
\sigma_{r r 1}=\left[\begin{array}{l}
C_{0}\left\{\begin{array}{l}
\left.\left(C_{11}-C_{12}\right)\left(1+\frac{u_{0}}{r}\right) \frac{\lambda q}{r} I_{2}(\lambda q r)\right\} \\
C_{11}\left(1+\frac{u_{0}}{r}\right)\left\langle\left(\lambda s_{i}\right)^{2} I_{1}\left(\lambda s_{i} r\right)-\frac{\lambda s_{i}}{r} I_{2}\left(\lambda s_{i} r\right)\right\rangle
\end{array}\right) \\
+C_{i}\left\{\begin{array}{l}
C_{12}\left(1+\frac{u_{0}}{r}\right)\left\langle\frac{\lambda s_{i}}{r} I_{2}\left(\lambda s_{i} r\right)\right\rangle \\
-C_{13}\left(1+\frac{\partial w_{0}}{\partial z}\right) \lambda^{2} k_{i} I_{1}\left(\lambda s_{i} r\right)
\end{array}\right.
\end{array}\right]
$$

$\times \cos \theta \cos \lambda z$

$$
\begin{aligned}
& {\left[C_{0}\left\{-\left(C_{11}-C_{12}\right)\left(1+\frac{u_{0}}{r}\right) \frac{\lambda q}{r} I_{2}(\lambda q r)\right\}\right.} \\
& \sigma_{\theta \theta 1}=\left\{\begin{array}{l}
C_{12}\left(1+\frac{u_{0}}{r}\right)\left\langle\left(\lambda s_{i}\right)^{2} I_{1}\left(\lambda s_{i} r\right)-\frac{\lambda s_{i}}{r} I_{2}\left(\lambda s_{i} r\right)\right\rangle \\
+C_{i 1}\left(1+\frac{u_{0}}{r}\right)\left\langle\frac{\lambda s_{i}}{r} I_{2}\left(\lambda s_{i} r\right)\right\rangle \\
-C_{13}\left(1+\frac{\partial w_{0}}{\partial z}\right) \lambda^{2} k_{i} I_{2}\left(\lambda s_{i} r\right)
\end{array}\right\}
\end{aligned}
$$

$\times \cos \theta \cos \lambda z$

$$
\sigma_{z z 1}=C_{i}\left[\begin{array}{l}
C_{13}\left\{\left(1+\frac{u_{0}}{r}\right)\left(\lambda s_{i}\right)^{2} I_{1}\left(\lambda s_{i} r\right)\right\} \\
-C_{33}\left\{\left(1+\frac{\partial w_{0}}{\partial z}\right) \lambda^{2} k_{i} I_{2}\left(\lambda s_{i} r\right)\right\}
\end{array}\right] \cos \theta \cos \lambda z
$$$$
\tau_{\theta z 1}=C_{55}\left[\begin{array}{l}
C_{0}\left(1+\frac{u_{0}}{r}\right) \lambda\left\{\frac{1}{r} I_{1}(\lambda q r)+\lambda q I_{2}(\lambda q r)\right\} \\
+C_{i}\left\{\left(1+\frac{u_{0}}{r}\right)+\left(1+\frac{\partial w_{0}}{\partial z}\right) k_{i}\right\} \frac{\lambda}{r} I_{1}\left(\lambda s_{i} r\right)
\end{array}\right]
$$

$\times \sin \theta \sin \lambda z$

$$
\tau_{z r 1}=-C_{55}\left[\begin{array}{l}
C_{0}\left(1+\frac{u_{0}}{r}\right) \frac{\lambda}{r} I_{1}(\lambda q r) \\
+C_{i}\left\{\left(1+\frac{u_{0}}{r}\right)+\left(1+\frac{\partial w_{0}}{\partial z}\right) k_{i}\right\} \lambda \\
\times\left\{\frac{1}{r} I_{1}\left(\lambda s_{i} r\right)+\lambda s_{i} I_{2}\left(\lambda s_{i} r\right)\right\}
\end{array}\right]
$$

$\times \cos \theta \sin \lambda z$

$$
\begin{aligned}
\tau_{r \theta 1}= & \frac{C_{11}-C_{12}}{2}\left[\begin{array}{l}
C_{0}\left\{-(\lambda q)^{2} I_{1}(\lambda q r)+\frac{2 \lambda q}{r} I_{2}(\lambda q r)\right\} \\
+C_{i}\left\{-\frac{2 \lambda s_{i}}{r} I_{2}\left(\lambda s_{i} r\right)\right\}
\end{array}\right] \\
& \times\left(1+\frac{u_{0}}{r}\right) \sin \theta \cos \lambda z
\end{aligned}
$$

Using the boundary condition on the free surface of the cylinder (Eq. (29)), we obtain the following equations.

$$
\begin{aligned}
& C_{0}\left[\left(C_{11}-C_{12}\right)\left(1+\frac{u_{0}}{r}\right) \frac{q}{a} I_{2}(\lambda q a)\right] \\
& +\sum_{i=1}^{2} C_{i}\left(1+\frac{u_{0}}{r}\right)\left\{\lambda s_{i}^{2} I_{1}\left(\lambda s_{i} a\right)-\frac{s_{i}}{a} I_{2}\left(\lambda s_{i} a\right)\right\} \\
& \quad=0 \\
& C_{0}\left(1+\frac{u_{0}}{r}\right)\left\{\frac{s_{i}}{a} I_{2}\left(\lambda s_{i} a\right)\right\} \\
& \left.\quad-\lambda q^{2} I_{1}(\lambda q a)+\frac{2 q}{a} I_{2}(\lambda q a)\right] \\
& \quad-\sum_{i=1}^{2} C_{i}\left[\frac{2 s_{i}}{a} I_{2}\left(\lambda s_{i} a\right)\right]=0 \\
& C_{0}\left[\left(1+\frac{u_{0}}{r}\right) \frac{1}{a} I_{1}(\lambda q a)\right] \\
& \left.\quad+\sum_{i=1}^{2} I_{1}\left(\lambda s_{i} a\right)\right\} \\
& \quad \times\left\{\frac { 1 } { a } I _ { 1 } \left(\lambda s_{i}\left(\{)+\lambda s_{i} I_{2}\left(\lambda s_{i} a\right)\right\}=0\right.\right.
\end{aligned}
$$

If we delete $u_{0} / r$ and $\partial w_{0} / \partial z$ in Eq. (55), we obtain the same equations as Eq. (28) in Kardomateas. ${ }^{3)}$ This means that the buckling load reported by Kardomateas is not an exact solution but an approximate one.

Equation (55) is simultaneous equations for $C_{0}, C_{1}$, and $C_{2}$. We equate the determinant of the coefficients of Eq. (55) as zero, and we obtain the eigenvalue, $p_{c r}$. The buckling stress $T_{c r}$ is calculated from Eq. (22).

\subsection{Numerical calculation procedure of buckling stress}

First, assume a value of $p_{c r}$, then calculate $s_{i}$ using Eqs. (23) and (36). Next, calculate $k_{i}$ using Eq. (38) and $q$ using Eq. (48). $s_{i}, k_{i}$, and $q$ are complex numbers in general. Substitute these numbers in Eq. (55), the determinant of the coefficients of Eq. (55) can be evaluated. Find the value of $p_{c r}$ with which the determinant becomes zero using "solver" of MS-Excel. 


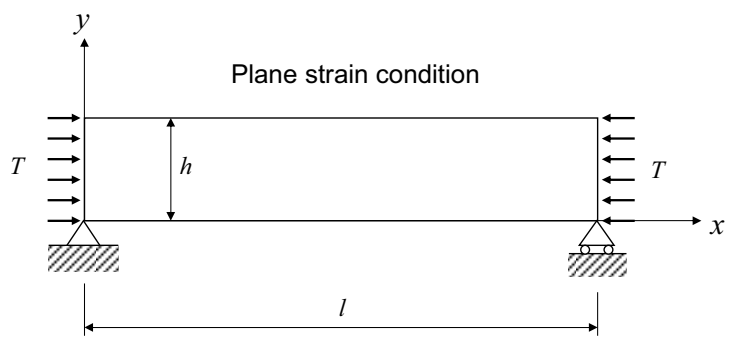

Fig. 4. Analysis model of 2D exact solution. ${ }^{2)}$

The values of the modified Bessel function of the first kind are calculated using the numerical integration of the following formula.

$$
I_{n}(z)=\frac{1}{\pi} \int_{0}^{\pi} e^{z \cos \theta} \cos (n \theta) \mathrm{d} \theta
$$

where $z$ is complex variable and $n$ is integer.

\section{Numerical Results}

\subsection{Effect of slenderness ratio on the buckling load}

Comparisons of the exact three-dimensional solution of the present paper, the approximate solution reported by Kardomateas $^{3)}$ and a two-dimensional exact solution reported by Chattopadhyay and $\mathrm{Gu}^{2)}$ are conducted below.

The relationship between the slenderness ratio $L^{\prime} / \rho$ and buckling load is calculated for the beam made of isotropic material. Since the solution by Chattopadhyay and $\mathrm{Gu}$ is based on a plane strain problem (see Fig. 4), the correction factor for Poisson's ratio is applied to the moment of inertia of the beam cross-section. The following equations are used to the calculate the slenderness ratio $L^{\prime} / \rho$.

-For the two-dimensional solution by Chattopadhyay and Gu:

$$
\frac{L^{\prime}}{\rho}=\frac{l}{h} \sqrt{12\left(1-v^{2}\right)}
$$

—For the three-dimensional solutions by Kardomateas and that in the present paper:

$$
\frac{L^{\prime}}{\rho}=4 \frac{L}{a}
$$

The buckling load results reported by Kardomateas are taken from Tables 1 and 2 of his paper. Figure 5 shows the comparison. The exact solutions for two-dimensional and three-dimensional elasticity are almost equal, but the buckling loads reported for the approximate solution of Kardomateas are larger than the exact solutions. The difference is large in the region of the small slenderness ratio.

\subsection{Effect of shear stiffness on buckling load}

The effect of shear stiffness on the buckling load is compared in this section. Two types of beam buckling load formula including transverse shear effect are proposed. ${ }^{1)}$ —Engesser's formula:

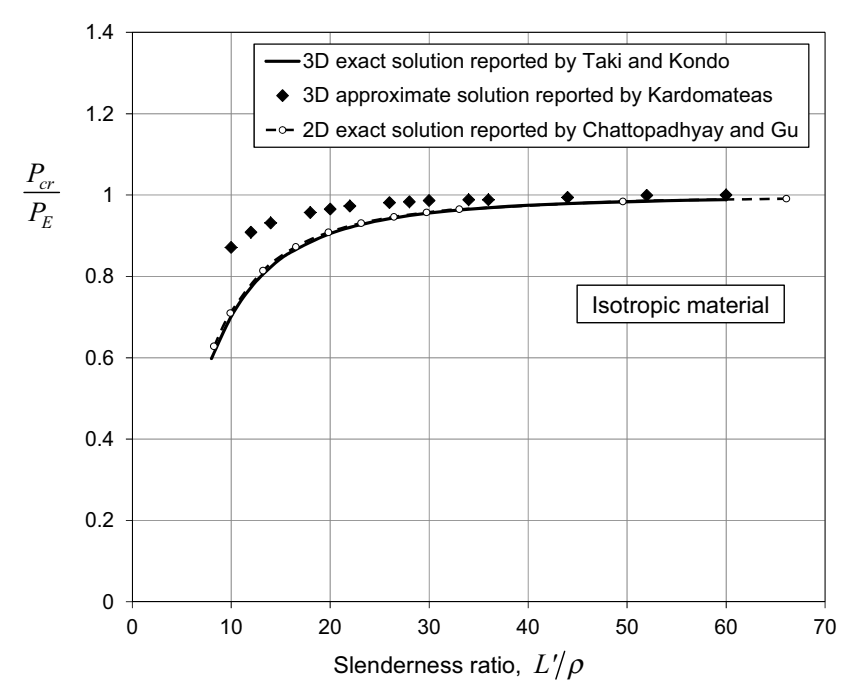

Fig. 5. Comparison of buckling loads-3D exact solution, 3D approximate solution and 2D exact solution.

Table 1. Material properties. ${ }^{3)}$

\begin{tabular}{lccc}
\hline & $\begin{array}{c}\text { Isotropic } \\
\text { material }\end{array}$ & $\begin{array}{c}\text { Material 1 } \\
\text { Glass/Epoxy }\end{array}$ & $\begin{array}{c}\text { Material 2 } \\
\text { Graphite/Epoxy }\end{array}$ \\
\hline$E_{L}(\mathrm{GPa})$ & 50 & 57 & 140 \\
$E_{T}(\mathrm{GPa})$ & 50 & 14 & 10 \\
$v_{L T}$ & 0.3 & 0.4 & 0.53 \\
$v_{T T}$ & 0.3 & 0.068 & 0.02 \\
$G_{L T}(\mathrm{GPa})$ & 19.23 & 5.7 & 5 \\
$C_{11}(\mathrm{GPa})$ & 67.31 & 14.76 & 10.22 \\
$C_{12}(\mathrm{GPa})$ & 28.85 & 1.648 & 0.4179 \\
$C_{13}(\mathrm{GPa})$ & 28.85 & 6.562 & 5.639 \\
$C_{33}(\mathrm{GPa})$ & 67.31 & 62.25 & 146.0 \\
$C_{55}(\mathrm{GPa})$ & 19.23 & 5.700 & 5.000 \\
\hline
\end{tabular}

$$
P_{E n}=\frac{P_{E}}{1+\frac{P_{E}}{G A_{e q}}}
$$

—Haringx's formula:

$$
P_{H}=\frac{\sqrt{1+\frac{4 P_{E}}{G A_{e q}}}-1}{\frac{2}{G A_{e q}}}
$$

where $P_{E}$ is Euler buckling load,

$$
P_{E}=\frac{\pi^{2} E I}{L^{\prime 2}}
$$

$I$ is the moment of inertia of the cross-section. $G A_{e q}=k G A$, $k$ is transverse shear correction-factor. $k=0.851$ for the circular cross-section, $k=5 / 6$ for the rectangular cross-section. ${ }^{10)} L^{\prime}$ is the effective length of the column.

Axial deformation is not considered in Eqs. (59) and (60).

As the slenderness ratio becomes smaller, the effect of axial deformation on the buckling load becomes significant. The buckling formulas, including both transverse shear and axial deformation, are as follows. 
Table 2. Comparison between Kardomateas' solution and that of the present paper.

\begin{tabular}{|c|c|c|c|c|c|c|c|c|c|c|c|c|c|}
\hline \multirow[b]{3}{*}{$L / a$} & \multirow[b]{3}{*}{$\begin{array}{c}E \\
(\mathrm{GPa}) \\
\end{array}$} & \multirow[b]{3}{*}{$\begin{array}{c}G \\
(\mathrm{GPa}) \\
\end{array}$} & \multirow[b]{3}{*}{$k$} & \multirow[b]{3}{*}{$P_{E} / E A$} & \multirow[b]{3}{*}{$P E / G A_{e q}$} & \multirow[b]{3}{*}{$\begin{array}{l}P_{E n} / P_{E} \\
\text { Eq. }(62)\end{array}$} & \multirow[b]{3}{*}{$\begin{array}{c}P_{H} / P_{E} \\
\text { Eq. (63) }\end{array}$} & \multicolumn{6}{|c|}{ Isotropic material } \\
\hline & & & & & & & & \multicolumn{3}{|c|}{ Kardomateas } & \multicolumn{3}{|c|}{ Taki and Kondo } \\
\hline & & & & & & & & $P_{E} / P_{K}$ & $P_{E n} / P_{K}$ & $P_{H} / P_{K}$ & $P_{E} / P_{T K}$ & $P_{E n} / P_{T K}$ & $P_{H} / P_{T K}$ \\
\hline 15 & 50 & 19.23 & 0.851 & 0.0027 & 0.0084 & 0.994 & 0.994 & 1.000 & 0.994 & 0.994 & 1.012 & 1.006 & 1.006 \\
\hline 13 & 50 & 19.23 & 0.851 & 0.0037 & 0.0112 & 0.992 & 0.993 & 1.001 & 0.993 & 0.994 & 1.015 & 1.008 & 1.008 \\
\hline 11 & 50 & 19.23 & 0.851 & 0.0051 & 0.0156 & 0.990 & 0.990 & 1.006 & 0.995 & 0.996 & 1.021 & 1.011 & 1.011 \\
\hline 9 & 50 & 19.23 & 0.851 & 0.0076 & 0.0233 & 0.984 & 0.985 & 1.012 & 0.996 & 0.997 & 1.032 & 1.016 & 1.016 \\
\hline 7 & 50 & 19.23 & 0.851 & 0.0126 & 0.0385 & 0.974 & 0.975 & 1.017 & 0.991 & 0.992 & 1.053 & 1.026 & 1.027 \\
\hline 5 & 50 & 19.23 & 0.851 & 0.0247 & 0.0754 & 0.949 & 0.954 & 1.036 & 0.983 & 0.988 & 1.105 & 1.048 & 1.054 \\
\hline \multirow[t]{3}{*}{3} & 50 & 19.23 & 0.851 & 0.0685 & 0.2094 & 0.859 & 0.889 & 1.101 & 0.946 & 0.979 & 1.294 & 1.112 & 1.150 \\
\hline & & & & & & & & \multicolumn{6}{|c|}{ Material 1-Glass/Epoxy } \\
\hline & & & & & & & & \multicolumn{3}{|c|}{ Kardomateas } & \multicolumn{3}{|c|}{ Taki and Kondo } \\
\hline$L / a$ & $\begin{array}{c}E \\
(\mathrm{GPa})\end{array}$ & $\begin{array}{c}G \\
(\mathrm{GPa})\end{array}$ & $k$ & $P_{E} / E A$ & $P E / G A_{e q}$ & $\begin{array}{l}P_{E n} / P_{E} \\
\text { Eq. }(62)\end{array}$ & $\begin{array}{c}P_{H} / P_{E} \\
\text { Eq. (63) }\end{array}$ & $P_{E} / P_{K}$ & $P_{E n} / P_{K}$ & $P_{H} / P_{K}$ & $P_{E} / P_{T K}$ & $P_{E n} / P_{T K}$ & $P_{H} / P_{T K}$ \\
\hline 15 & 57 & 5.7 & 0.851 & 0.0027 & 0.0322 & 0.971 & 0.972 & 1.017 & 0.988 & 0.989 & 1.034 & 1.005 & 1.006 \\
\hline 13 & 57 & 5.7 & 0.851 & 0.0037 & 0.0429 & 0.962 & 0.964 & 1.022 & 0.983 & 0.985 & 1.046 & 1.006 & 1.008 \\
\hline 11 & 57 & 5.7 & 0.851 & 0.0051 & 0.0599 & 0.948 & 0.950 & 1.031 & 0.977 & 0.980 & 1.064 & 1.008 & 1.011 \\
\hline 9 & 57 & 5.7 & 0.851 & 0.0076 & 0.0895 & 0.923 & 0.929 & 1.047 & 0.967 & 0.973 & 1.095 & 1.011 & 1.018 \\
\hline 7 & 57 & 5.7 & 0.851 & 0.0126 & 0.1479 & 0.878 & 0.892 & 1.080 & 0.949 & 0.964 & 1.157 & 1.017 & 1.033 \\
\hline 5 & 57 & 5.7 & 0.851 & 0.0247 & 0.2899 & 0.783 & 0.821 & 1.159 & 0.908 & 0.952 & 1.307 & 1.024 & 1.073 \\
\hline \multirow[t]{3}{*}{3} & 57 & 5.7 & 0.851 & 0.0685 & 0.8054 & 0.556 & 0.670 & 1.439 & 0.800 & 0.964 & 1.839 & 1.022 & 1.231 \\
\hline & & & & & & & & \multicolumn{6}{|c|}{ Material 2-Graphite/Epoxy } \\
\hline & & & & & & & & & ardomate & & & ki and Kon & \\
\hline$L / a$ & $\begin{array}{c}E \\
(\mathrm{GPa})\end{array}$ & $\begin{array}{c}G \\
(\mathrm{GPa})\end{array}$ & $k$ & $P_{E} / E A$ & $P E / G A_{e q}$ & $\begin{array}{l}P_{E n} / P_{E} \\
\text { Eq. }(62)\end{array}$ & $\begin{array}{c}P_{H} / P_{E} \\
\text { Eq. (63) }\end{array}$ & $P_{E} / P_{K}$ & $P_{E n} / P_{K}$ & $P_{H} / P_{K}$ & $P_{E} / P_{T K}$ & $P_{E n} / P_{T K}$ & $P_{H} / P_{T K}$ \\
\hline 15 & 140 & 5 & 0.851 & 0.0027 & 0.0902 & 0.919 & 0.925 & 1.05 & 0.965 & 0.971 & 1.091 & 1.003 & 1.009 \\
\hline 13 & 140 & 5 & 0.851 & 0.0037 & 0.1201 & 0.895 & 0.905 & 1.065 & 0.953 & 0.963 & 1.121 & 1.003 & 1.014 \\
\hline 11 & 140 & 5 & 0.851 & 0.0051 & 0.1677 & 0.859 & 0.875 & 1.093 & 0.939 & 0.957 & 1.169 & 1.004 & 1.023 \\
\hline 9 & 140 & 5 & 0.851 & 0.0076 & 0.2506 & 0.803 & 0.832 & 1.138 & 0.913 & 0.947 & 1.251 & 1.004 & 1.041 \\
\hline 7 & 140 & 5 & 0.851 & 0.0126 & 0.4142 & 0.710 & 0.765 & 1.228 & 0.872 & 0.939 & 1.414 & 1.004 & 1.082 \\
\hline 5 & 140 & 5 & 0.851 & 0.0247 & 0.8118 & 0.553 & 0.659 & 1.449 & 0.801 & 0.954 & 1.806 & 0.998 & 1.189 \\
\hline 3 & 140 & 5 & 0.851 & 0.0685 & 2.2551 & 0.305 & 0.485 & 2.227 & 0.678 & 1.081 & 3.203 & 0.976 & 1.554 \\
\hline
\end{tabular}

—Engesser's formula ${ }^{11,12)}$ :

$$
\begin{aligned}
& \left(\frac{P_{E}}{E A}\right)^{2}\left(\frac{P_{E n}}{P_{E}}\right)^{3}-2\left(\frac{P_{E}}{E A}\right)\left(\frac{P_{E n}}{P_{E}}\right)^{2} \\
& \quad+\left(1+\frac{P_{E}}{E A}+\frac{P_{E}}{G A_{e q}}\right)\left(\frac{P_{E n}}{P_{E}}\right)-1=0
\end{aligned}
$$

-Haringx's formula ${ }^{1)}$ :

$$
P_{H}=\frac{\sqrt{1+4\left(\frac{P_{E}}{G A_{e q}}-\frac{P_{E}}{E A}\right)}-1}{2\left(\frac{P_{E}}{G A_{e q}}-\frac{P_{E}}{E A}\right)} P_{E}
$$

$P_{E} / E A$ is a parameter of the axial stiffness and $P_{E} / G A_{e q}$ is a parameter of the shear stiffness. These parameters for a circular cross-section beam, with one end fixed and the other end free (Fig. 1), are expressed as follows.

$$
\frac{P_{E}}{E A}=\frac{\pi^{2} a^{2}}{16 L^{2}}, \quad \frac{P_{E}}{G A_{e q}}=\frac{\pi^{2} E a^{2}}{16 k G L^{2}}
$$

\subsubsection{Discussion on Table 1 of Kardomateas' paper ${ }^{3)}$}

Kardomateas analyzed the buckling loads of beams made of three materials: isotropic material, glass/epoxy and graphite epoxy. He compared the buckling loads with Engesser's and Haringx's formulas, Eqs. (59) and (60). Because the comparison includes beams with a small slenderness ratio, it is necessary to use Eqs. (62) and (63) rather than Eqs. (59) and (60). Comparisons between three-dimensional elasticity solutions and Eqs. (62) and (63) are conducted using the material properties shown in Table 1 . The results are shown in Table 2 and Figs. 6 to 8.

As Kardomateas stated, his solution (approximate solution) is in better agreement with Haringx's formula than Engesser's formula, while the exact solution of the present paper is in better agreement with Engesser's formula.

It is noteworthy that the approximate solution for the isotropic material beam is in better agreement with both beam formulas than the exact solution (Fig. 6). The reason for this is that the effect of shear is small and the effect of slenderness ratio is significant for isotropic material.

For orthotropic materials, which are soft in transverse shear, the effect of shear deformation becomes more dominant than axial deformation, consequently, agreement between the exact solution and Engesser's formula is better than that with isotropic material. 
Trans. Japan Soc. Aero. Space Sci., Vol. 60, No. 1, 2017

Table 3. Comparison of buckling loads, and effect of transverse shear stiffness.

\begin{tabular}{|c|c|c|c|c|c|c|c|}
\hline \multirow{2}{*}{ Slenderness ratio } & \multirow{2}{*}{$G_{L T} / E_{L}$} & \multirow{2}{*}{$P_{E} / E A$} & \multirow{2}{*}{$P_{E} / G A_{e q}$} & \multicolumn{4}{|c|}{$P_{c r} / P_{E}$} \\
\hline & & & & Engesser Eq. (59) & Haringx Eq. (60) & Taki and Kondo & Kardomateas \\
\hline 40 & 0.1 & 0.0062 & 0.0725 & 0.932 & 0.936 & 0.927 & 0.962 \\
\hline 40 & 0.08 & 0.0062 & 0.0906 & 0.917 & 0.923 & 0.912 & 0.952 \\
\hline 40 & 0.06 & 0.0062 & 0.1208 & 0.892 & 0.902 & 0.888 & 0.937 \\
\hline 40 & 0.05 & 0.0062 & 0.1450 & 0.873 & 0.886 & 0.869 & 0.925 \\
\hline 40 & 0.04 & 0.0062 & 0.1812 & 0.847 & 0.865 & 0.843 & 0.908 \\
\hline 40 & 0.03 & 0.0062 & 0.2416 & 0.805 & 0.833 & 0.803 & 0.881 \\
\hline 40 & 0.025 & 0.0062 & 0.2899 & 0.775 & 0.810 & 0.773 & 0.860 \\
\hline 40 & 0.02 & 0.0062 & 0.3624 & 0.734 & 0.780 & 0.733 & 0.831 \\
\hline 40 & 0.0175 & 0.0062 & 0.4142 & 0.707 & 0.760 & 0.707 & 0.812 \\
\hline 40 & 0.015 & 0.0062 & 0.4832 & 0.674 & 0.737 & 0.674 & 0.787 \\
\hline
\end{tabular}

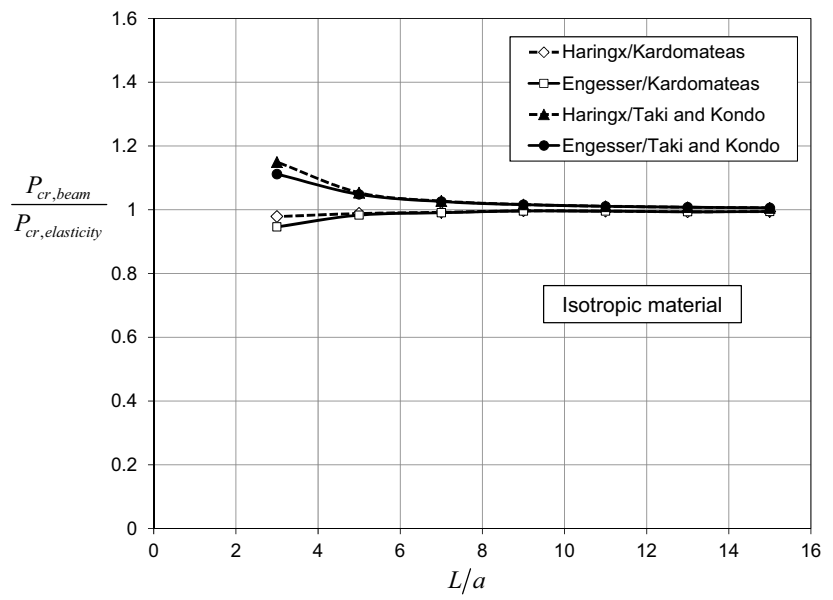

Fig. 6. Comparison of buckling loads of isotropic material-3D exact solution, 3D approximate solution and beam formulas.

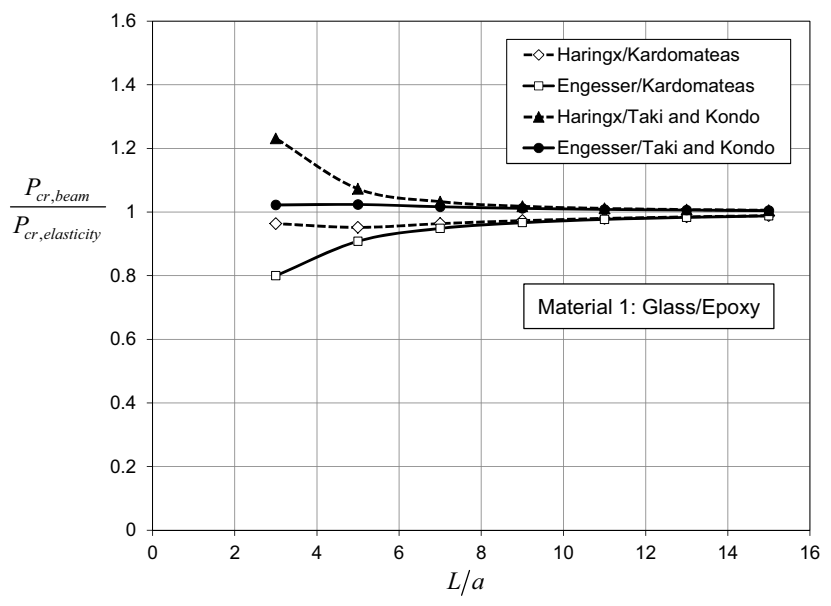

Fig. 7. Comparison of buckling loads of material $1-3 \mathrm{D}$ exact solution, 3D approximate solution and beam formulas.

\subsubsection{Effect of transverse shear stiffness}

The results of Figs. 6 to 8 include both shear and axial deformation effects. It is necessary to eliminate the effect of axial deformation in order to evaluate the correctness of the beam formulas. A beam with a high slenderness ratio and weak shear stiffness is analyzed. It is assumed that the slenderness ratio is 40 and that the hypothetical orthotropic material has the following material properties.

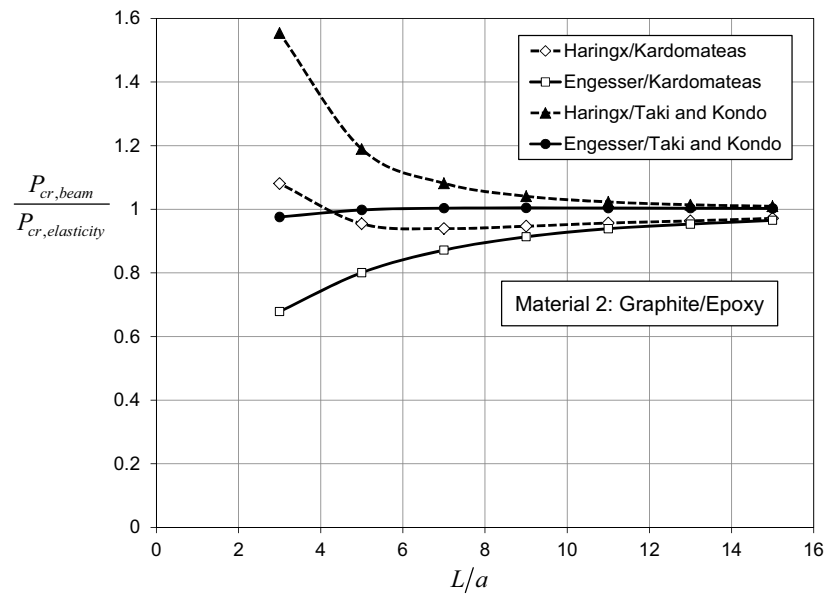

Fig. 8. Comparison of buckling loads of material 2-3D exact solution, 3D approximate solution and beam formulas.

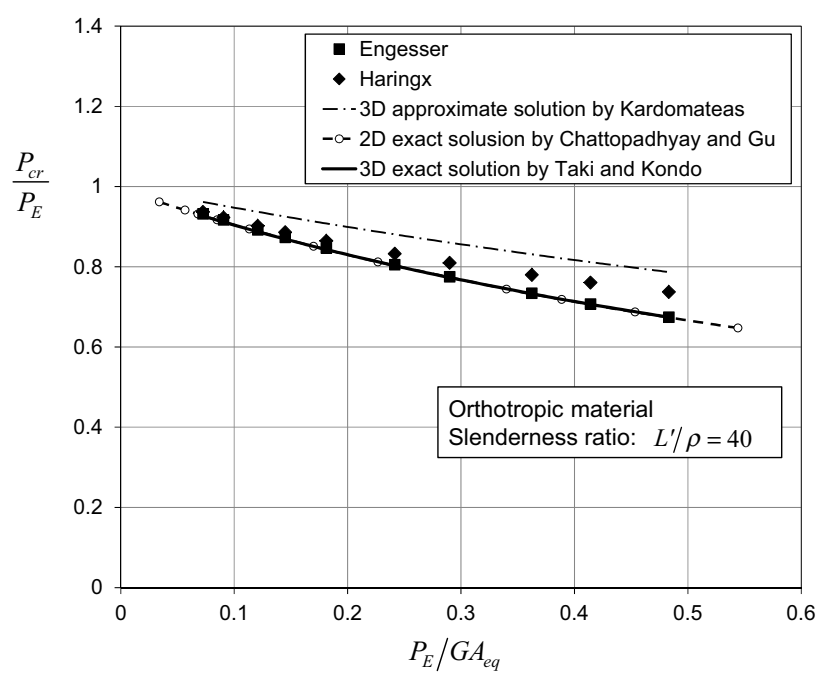

Fig. 9. Comparison of buckling loads, and effect of transverse shear stiffness.

$E_{L}=100 \mathrm{GPa}, \quad E_{T}=10 \mathrm{GPa}, \quad v_{L T}=0.3, \quad v_{T T}=0.3$

$G_{L T}=1.5-10 \mathrm{GPa}$

The results are shown in Table 3 and Fig. 9. The values of $P_{c r} / P_{E}$ using Engesser and Haringx formulas, as shown in Table 3, are based on Eqs. (59) and (60), respectively, and 
the values are almost equal to the values from Eqs. (62) and (63) because the effect of axial deformation is negligible. The values obtained using Engesser's formula are on the curve of three-dimensional and two-dimensional exact solutions. This result confirms that Engesser's formula is the correct beam formula, and not Haringx's formula. The threedimensional approximate solution reported by Kardomateas is closer to Haringx's formula, and this is the reason why Kardomateas erroneously concluded that Haringx formula is the correct formula.

\section{Conclusions}

(1) An exact three-dimensional elasticity solution for buckling of a beam with a circular cross-section is derived. The buckling load derived using the three-dimensional solution is in good agreement with the two-dimensional solution reported by Chattopadhyay and $\mathrm{Gu}$.

(2) The exact three-dimensional elasticity solution confirms that Engesser's formula is correct for the buckling load of beam with a transverse shear effect. Haringx's formula should not be applied to beams.

(3) It is shown that Kardomateas' solution ${ }^{3)}$ is an approximate solution.

\section{References}

1) Timoshenko, S. P. and Gere, J. M.: Theory of Elastic Stability, Second
Edition, Dover Publications, Inc., Mineola, New York, 2011.

2) Chattopadhyay, A. and Gu, H.: Exact Elasticity Solution for Buckling of Composite Laminates, Compos. Struct., 34 (1996), pp. 291-299.

3) Kardomateas, G. A.: Three-Dimensional Elasticity Solution for the Buckling of Transversely Isotropic Rods: The Euler Load Revisited, J. Appl. Mech., 62 (1995), pp. 346-355.

4) Bažant, Z. P.: Shear Buckling of Sandwich, Fiber Composite and Lattice Columns, Bearings, and Helical Springs: Paradox Resolved, J. Appl. Mech., 70 (2003), pp. 70-83.

5) Bažant, Z. P. and Beghini, A.: Which Formulation Allows Using a Constant Shear Modulus for Small-Strain Buckling of Soft-Core Sandwich Structures? J. Appl. Mech., 72 (2005), pp. 785-787.

6) Bažant, Z. P. and Beghini, A.: Stability and Finite Strain of Homogenized Structures Soft in Shear: Sandwich or Fiber Composites, and Layered Bodies, Int. J. Solids Struct., 43 (2006), pp. 1571-1593.

7) Attard, M. M.: Global Buckling Experiments on Sandwich Columns with Soft Shear Cores, Electr. J. Struct. Eng., 11 (2011), pp. 21-31.

8) Blaauwendraad, J.: Shear in Structural Stability: On the Engesser-Haringx Discord, J. Appl. Mech., 77 (2010), pp. 031005-1-8.

9) Taki, T.: Note on Compression Buckling of Shearable Beam, Proc. of 56th JSASS/JSME/JAXA Structures Conference, 2014, pp. 60-62 (in Japanese).

10) Washizu, K.: Variational Methods in Elasticity and Plasticity, Pergamon Press, Oxford, 1968.

11) Iwakura, T. and Kuranishi, S.: How Much Contribution Does the Shear Deformation Have in a Beam Theory? Proc. JSCE, 344, I-1 (1984), pp. 141-151.

12) Kondo, K.: Buckling of Extensible Shearable Elastica, Proc. of 54th JSASS/JSME/JAXA Structures Conference, 2008, pp. 61-63 (in Japanese).
T. Ogasawara Associate Editor 